Article

\title{
CmMYB19 Over-Expression Improves Aphid Tolerance in Chrysanthemum by Promoting Lignin Synthesis
}

\author{
Yinjie Wang ${ }^{\dagger}$, Liping Sheng ${ }^{\dagger}$, Huanru Zhang, Xinping Du, Cong An, Xiaolong Xia, Fadi Chen, \\ Jiafu Jiang and Sumei Chen * \\ College of Horticulture, Nanjing Agricultural University, Nanjing 210095, China; \\ 2013204034@njau.edu.cn (Y.W.); 2013104116@njau.edu.cn (L.S.); zhanghuanru@sina.cn (H.Z.); \\ 2013104106@njau.edu.cn (X.D.); frozen_water@163.com (C.A.); xiaxiaolongyx@163.com (X.X.); \\ chenfd@njau.edu.cn (F.C.); jiangjiafu@njau.edu.cn (J.J.) \\ * Correspondence: chensm@njau.edu.cn; Tel.: +86-25-8439-9670; Fax: 86-25-8439-5266 \\ + These authors contributed equally to this work.
}

Academic Editors: Massimo Maffei and Francesca Barbero

Received: 18 December 2016; Accepted: 7 March 2017; Published: 12 March 2017

\begin{abstract}
The gene encoding the MYB (v-myb avian myeloblastosis vira 1 oncogene homolog) transcription factor CmMYB19 was isolated from chrysanthemum. It encodes a 200 amino acid protein and belongs to the R2R3-MYB subfamily. CmMYB19 was not transcriptionally activated in yeast, while a transient expression experiment conducted in onion epidermal cells suggested that the CmMYB19 product localized to the nucleus. CmMYB19 transcription was induced by aphid (Macrosiphoniella sanborni) infestation, and the abundance of transcript was higher in the leaf and stem than in the root. The over-expression of CmMYB19 restricted the multiplication of the aphids. A comparison of transcript abundance of the major genes involved in lignin synthesis showed that CmPAL1 (phenylalanine ammonia lyase 1), $\mathrm{CmC4H}$ (cinnamate4 hydroxylase), Cm4CL1 (4-hydroxy cinnamoyl CoA ligase 1), CmHCT (hydroxycinnamoyl CoA-shikimate/quinate hydroxycinnamoyl transferase), $\mathrm{CmC} 3 \mathrm{H1}$ (coumarate3 hydroxylase1), CmCCoAOMT1 (caffeoyl CoA O-methyltransferase 1) and CmCCR1 (cinnamyl CoA reductase1) were all upregulated, in agreement with an increase in lignin content in CmMYB19 over-expressing plants. Collectively, the over-expression of CmMYB19 restricted the multiplication of the aphids on the host, mediated by an enhanced accumulation of lignin.
\end{abstract}

Keywords: Chrysanthemum morifolium; expression analysis; aphid; transgenic plants; lignin

\section{Introduction}

The large v-myb avian myeloblastosis vira 1 oncogene homolog (MYB) transcription factor family is functionally highly diverse. The defining characteristic of these proteins is the presence of the so-called MYB domain, which provides the basis of its DNA binding ability [1]; it comprises some 52 residues which adopt a helix-turn-helix conformation [2]. The MYB proteins have been sub-classified into four types, the largest of which is the R2R3-MYB subfamily [3].

MYB transcription factors are important in the regulation of lignin synthesis, a wellcharacterized pathway which encompasses the enzymes phenylalanine ammonia lyase (PAL), cinnamate 4-hydroxylase $(\mathrm{C} 4 \mathrm{H})$, 4-hydroxy cinnamoyl CoA ligase (4CL), hydroxycinnamoyl CoA-shikimate/quinate hydroxycinnamoyltransferase (HCT), p-coumarate 3-hydroxylase $(\mathrm{C} 3 \mathrm{H})$, caffeoyl CoA O-methyltransferase (CCoAOMT), cinnamoyl CoA reductase (CCR), ferulate 5-hydroxylase ( $\mathrm{F} 5 \mathrm{H})$, caffeic acid/5-hydroxyferulic acid O-methyltransferase (COMT) 
and cinnamyl alcohol dehydrogenase (CAD) [4]. A. thaliana MYB46 is not only a key regulator of lignin synthesis, but also activates the entire process of secondary cell wall formation [5]. The over-expression of either AtMYB58 or AtMYB63 upregulates a number of lignin synthesis genes, leading to the ectopic deposition of lignin [4]. Similarly, in poplar, the over-expression of PtoMYB216 activates the transcription of certain lignin synthesis genes, resulting in ectopic lignin deposition [6]. In contrast, the heterologous transcription of PdMYB221 in A. thaliana reduces the cell wall thickness of fibers and vessels, thereby negatively affecting secondary wall formation in the stem [7]. MYB transcription factors typically recognize certain AC-rich cis elements ([ACC (T/A) ACC]), which are particularly common in the promoters of $P A L, 4 C L, C C R$ and $C A D$ [8], thereby regulating the lignin biosynthesis.

Aphid is an important insect for crops, which deprives the plant of nutrients and is the vector of certain viruses that further compromise productivity [9]. Plants have evolved a number of strategies to cope with aphid infestation, of which constitutively expressed tolerance is an effective defense in response to insect feeding, which resulted from the presence of host-synthesized compounds or structures, the former including toxins such as tannic acid or mustard oil, and the latter including physical barriers formed by lignin, trichomes and surface wax [10]. We supposed that MYBs involved in lignin biosynthesis might regulate constitutive defense to insect feeding by strengthening the physical barrier.

Chrysanthemum (Chrysanthemum morifolium) is one of the most valuable ornamental species. One of the major constraints to the quality of the commercial product is the aphid Macrosiphoniella sanborni, which greatly hampers chrysanthemum growth and yield [9]. R2R3-MYB genes participate in a wide range of biological processes [11], which includes a few examples of involvement in the response to insect feeding. For example, the Arabidopsis thaliana transcription factor MYB102 restricts the development of Pieris rapae caterpillars [12], while MYB44 activity has been associated with tolerance to both the green peach aphid and the diamondback Moth in Arabidopsis [13]. To date, no MYB transcription factors have been associated with the response of chrysanthemum to aphid feeding. Here, a description is given of the isolation of the chrysanthemum MYB gene CmMYB19, the product of which is associated with lignin synthesis and the defense against aphid feeding.

\section{Results}

\subsection{Isolation and Phylogenetic Analysis of the CmMYB19 cDNA Sequence}

The $994 \mathrm{nt}$ CmMYB19 (KT763375) sequence isolated from NX included a $600 \mathrm{nt}$ ORF. The predicted gene encoded a 200 residue polypeptide containing two conserved MYB domains, on the basis of which it was assigned as a member of the R2R3-MYB subfamily (Figure 1), with a similarity ranging from $31.85 \%$ (AtMYB19) to $19.30 \%$ (AtMYB103). An alignment of the deduced amino acid sequences of CmMYB19 with $A$. thaliana R2R3-MYBs showed that CmMYB19 were included in a lignin biosynthesis group or wall deposition, and its most similar homolog was AtMYB19 (Figure 2).

\subsection{Transcriptional Activation and Sub-Cellular Localization}

To characterize the transcriptional activation of CmMYB19. Yeast cells harboring either an empty pGBKT7 or the pGBKT7-CmMYB19 construct were incapable of growing on SD/-His-Ade medium, whereas those harboring pCL1 grew well (Figure 3). Thus, the indication is that CmMYB19 expresses no transcriptional activation in yeast cells. In transiently transformed onion epidermal cells, the control transgene (p35S::GFP) produced a diffuse GFP signal, but the GFP activity induced by the p35S::GFP-CmMYB19 transgene was restricted to the nucleus (Figure 4). Thus, in vivo, CmMYB19 product is likely localized to the nucleus.

\subsection{Transcription of CmMYB19 in the Chrysanthemum Plant}

CmMYB19 transcripts was detectable in the root, stem and leaf of NX plants. The highest abundance was observed in the leaf and the lowest in the root (Figure 5). The pattern of transcription 
as induced by aphid infestation differed from that induced by the mock puncture treatment. CmMYB19 was upregulated by the presence of aphids at $0 \mathrm{~h}, 6 \mathrm{~h}, 9 \mathrm{~h}$ and $24 \mathrm{~h}$, but was downregulated at $3 \mathrm{~h}$ and $12 \mathrm{~h}$; meanwhile, the mock puncture treatment lowered the transcript abundance after $3 \mathrm{~h}, 6 \mathrm{~h}$ and $12 \mathrm{~h}$, but had no effect at the other sampling times (Figure 6).

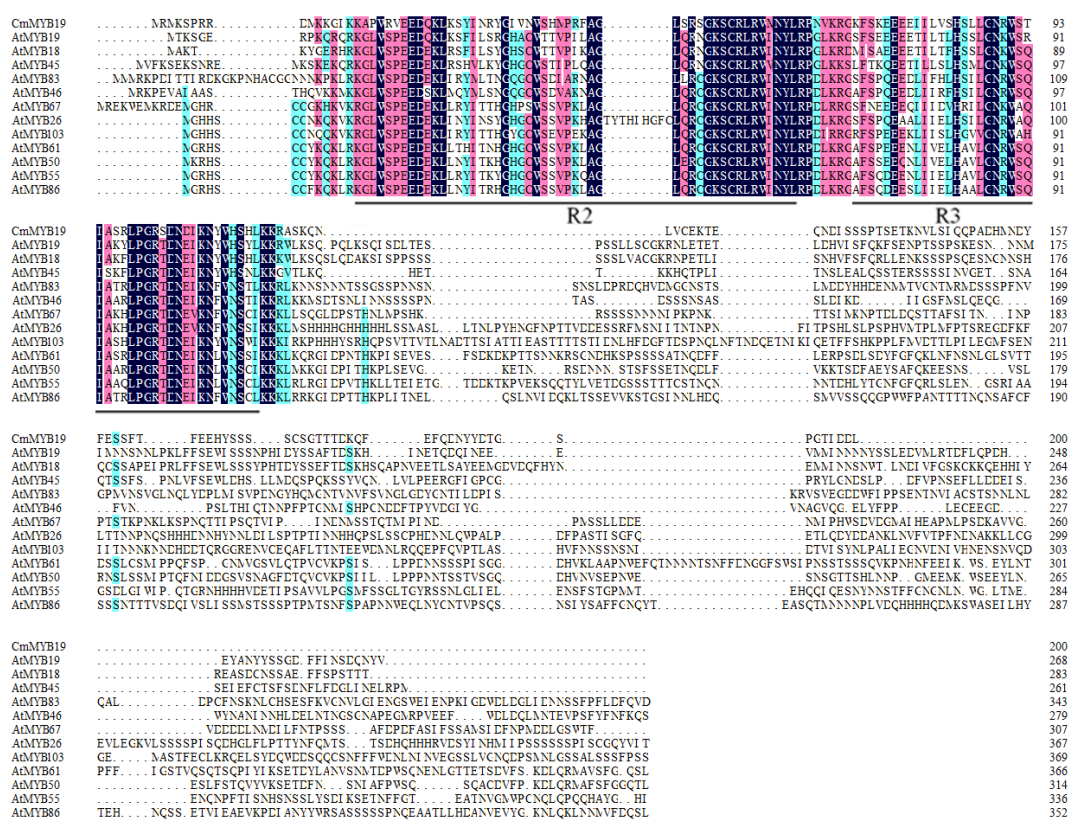

Figure 1. Alignment of the putative CmMYB19 protein sequence with those of R2R3 MYBs from lignin biosynthesis or wall deposition group in Arabidopsis. The two MYB domains are indicated by lines drawn below the alignment.

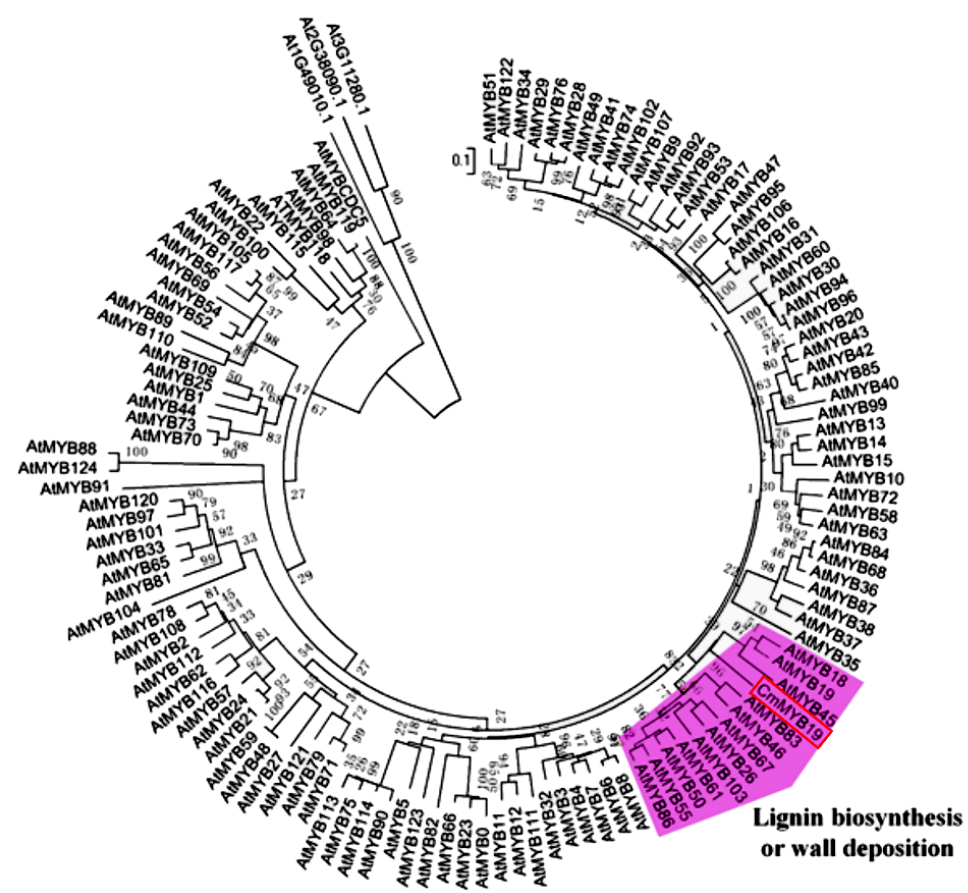

Figure 2. Phylogenetic analysis of CmMYB19 (boxed) and A. thaliana R2R3-MYB members. The alignment was generated by ClustalW (available on: http://www.ebi.ac.uk/Tools/msa/ clustalw2/), and the neighbor-joining tree constructed using MEGA 5 (available on: http:/ www. megasoftware.net) (1000 bootstrap replicates). 


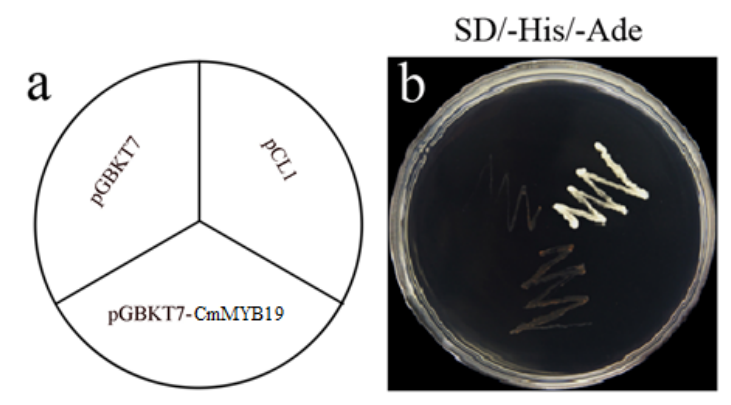

Figure 3. Transcription activation of CmMYB19 in yeast. (a) the orientation of the plate. (b) the growth of transformed yeast cells. Cells harboring pCL1 were able to grow on the SD/-His-Ade medium (SD, Synthetic Dropout Media), while those containing pGBKT7 could not.

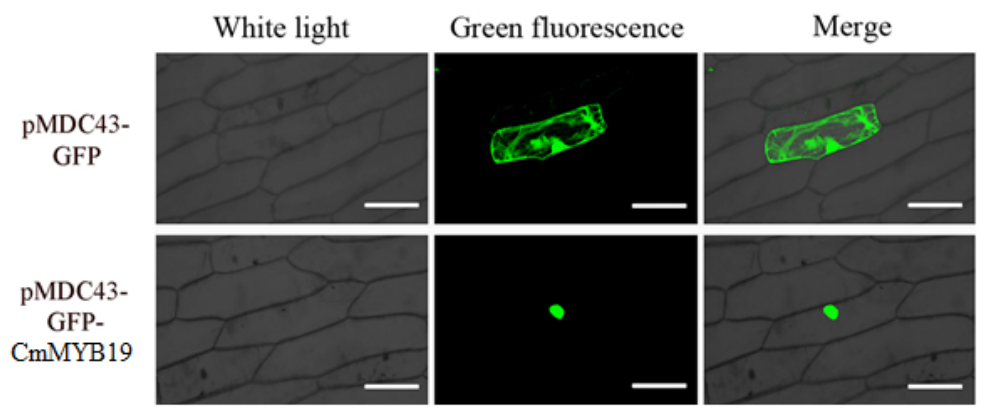

Figure 4. Sub-cellular localization of CmMYB19 expression in transiently transformed onion epidermal cells. Bar: $100 \mu \mathrm{m}$.

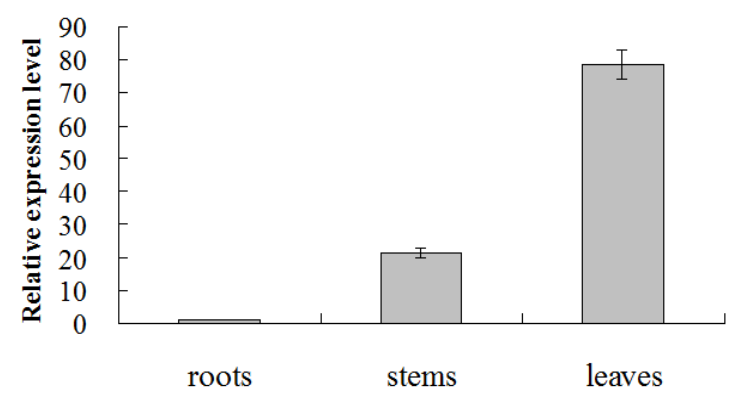

Figure 5. Transcriptional changes of CmMYB19 in various organs of the chrysanthemum plant, as assayed by qRT-PCR.

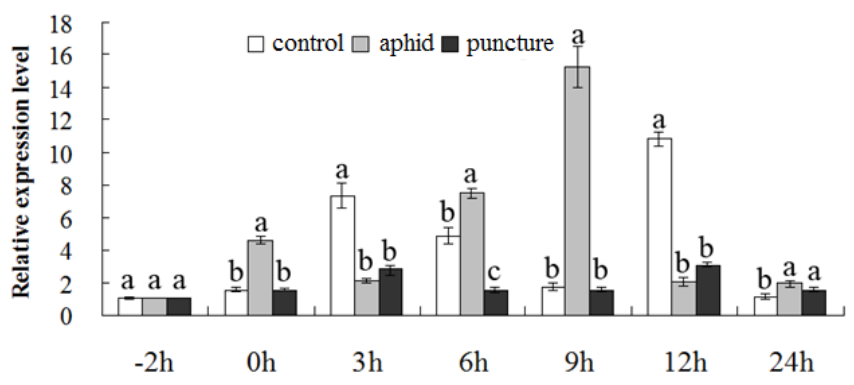

Figure 6. Transcriptional changes of CmMYB19 following aphid infestation or mock puncture treatment, as assayed by qRT-PCR. At each time point, different letters $(a, b, c)$ designate significantly different expression levels between control, aphid infestation and puncture $(p<0.05)$. 


\subsection{CmMYB19 Over-Expression Enhanced Aphid Tolerance}

Putative CmMYB19 overexpressing lines were selected on cultural medium supplemented with hygromycin (Supplemental Figures S1 and S2). The overexpressing lines were verified by amplification of fragment of $\mathrm{Hyg}^{r}$ gene, the bands presented in the transgenic plants while absent in non-transgenic plants (Figure 7a), and CmMYB19 transcript abundance was clearly higher than in non-transgenic JB in both of the two independent CmMYB19 over-expression lines (CmMYB19-ox-1 and CmMYB19-ox-2) (Figure $7 b$ ). Over the course of the 21 days when aphid numbers were monitored, the aphids multiplied more freely on the non-transgenic plants than on either -ox-1 or -ox-2, whereas, on the non-transgenic plants, the aphids were distributed widely throughout the plants (Figure 7c,d). On the non-transgenic plants, aphid numbers multiplied rapidly from 7 DAI, reaching a mean of 142.4 per plant by 21 DAI (Figure 7c), equivalent to an MR of 28.5 (Table 1). In contrast, on -ox-1, the mean number of aphids present at $21 \mathrm{DAI}$ was only $83.9(\mathrm{MR}=16.8, \mathrm{IR}=41.1)$, and the equivalents for -ox-2 were, respectively, 98.4, 19.7 and 30.9 (Figure 7c, Table 1). Thus, the over-expression of CmMYB19 clearly inhibited the feeding and reproduction of the aphids.

Table 1. Aphid multiplication on CmMYB19 over-expressing and non-transgenic plants

\begin{tabular}{cccc}
\hline \multicolumn{2}{c}{ Plants } & MR & IR \\
\hline \multicolumn{2}{c}{ Non-transgenic plants } & $28.48 \pm 0.262^{\mathrm{a}}$ & 0 \\
\multirow{2}{*}{ Transgenic plants } & CmMYB19-ox-1 & $16.78 \pm 0.548^{\mathrm{c}}$ & 41.08 \\
& CmMYB19-ox-2 & $19.68 \pm 0.776^{\mathrm{b}}$ & 30.90 \\
\hline
\end{tabular}

* different letters $\left({ }^{\mathrm{a}},{ }^{\mathrm{b}},{ }^{\mathrm{c}}\right)$ represent significantly different aphid multiplication between non-transgenic and CmMYB19 overexpressing plants.

a

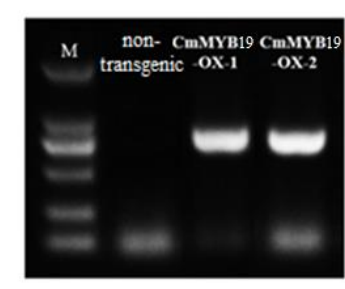

d

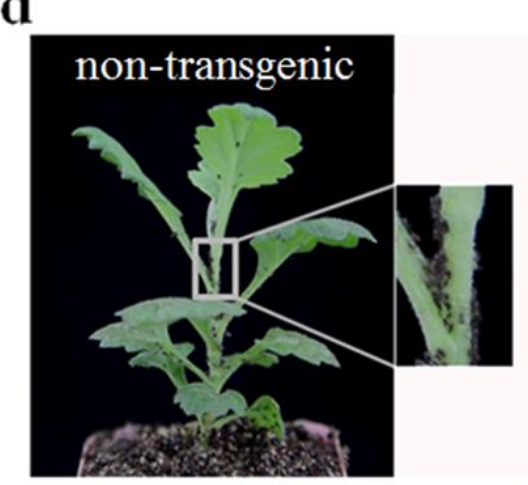

$\mathrm{b}$
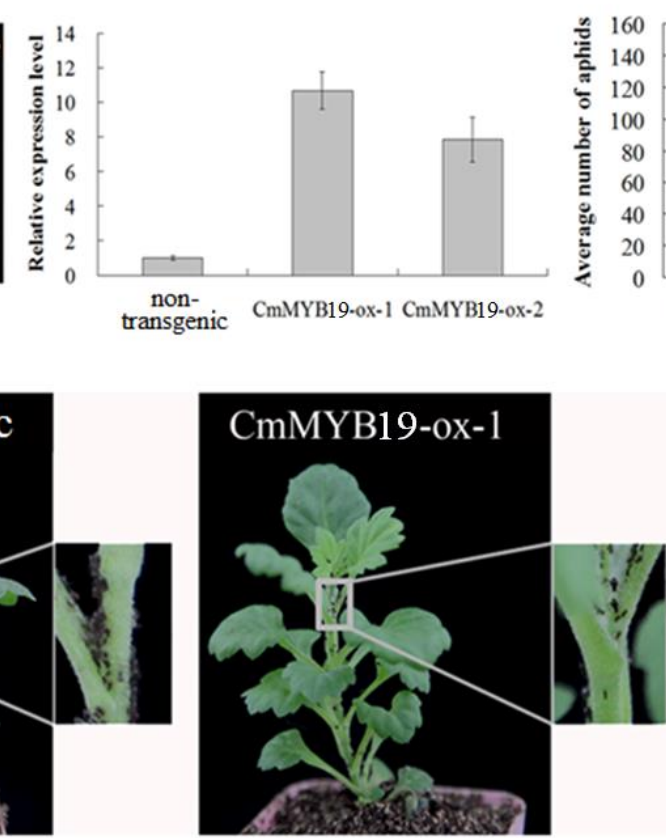

c
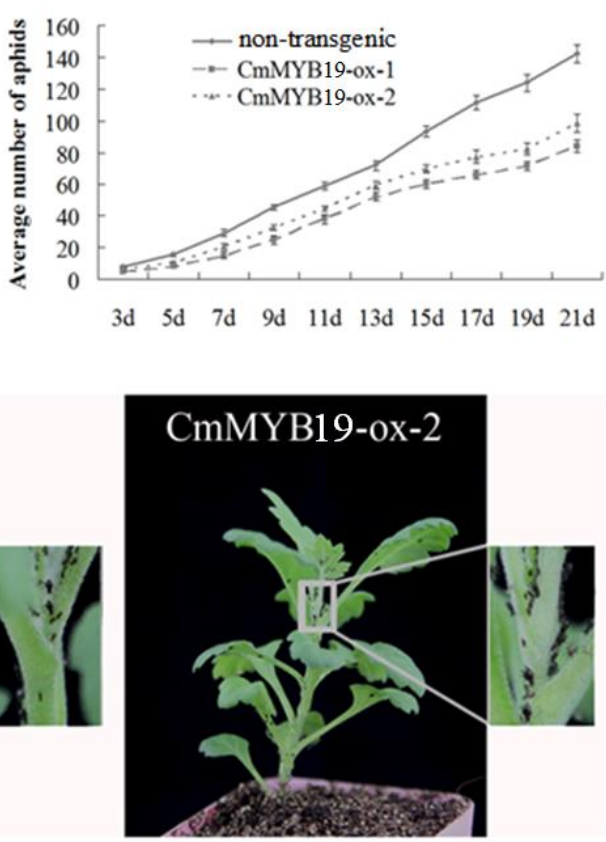

Figure 7. Transgenic chrysanthemum lines over-expressing CmMYB19 inhibit the multiplication of M. sanbourni aphids. (a) Amplification of fragment of $H_{y g}{ }^{r}$ gene. M, molecular marker, non-transgenic, CmMYB19-ox-1, CmMYB19-ox-2 were non-transgenic plants and CmMYB19 overexpressing plants, respectively; (b) CmMYB19 transcript abundance in CmMYB19 over-expressing and non-transgenic plants; (c) the number of aphids present on CmMYB19 over-expressing and non-transgenic plants measured at 3-21 d (days) after infestation; and (d) differential proliferation of aphids between CmMYB19 over-expressing and non-transgenic plants. 
2.5. Lignin Content and the Transcript Abundance of Genes Involved in Lignin Synthesis in CmMYB19 Over-Expressors

The lignin content of both -ox lines was 2.46 and 1.57 fold that of WT JB (Figure 8). Among the lignin synthesis gene surveyed, CmPAL1, CmC4H,Cm4CL1, CmHCT, CmC3H1, CmCCoAOMT1 and CmCCR1 were all more strongly transcribed in the -ox lines, while the abundance of CmF5H1, CmCOMT and CmCAD6 transcript was not markedly altered (Figure 9). The conclusion was that CmMYB19 over-expression promoted the accumulation of lignin by upregulating the transcription of some of the genes involved in lignin synthesis.

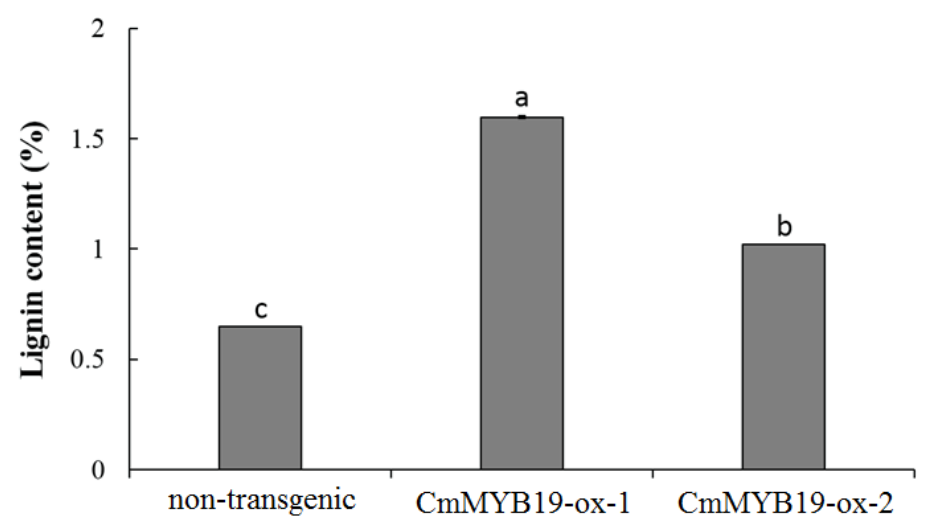

Figure 8. Lignin content determined in CmMYB19 over-expressing and non-transgenic plants. a, b, c represent significantly different expression levels between non-transgenic and CmMYB19 overexpressing plants $(p<0.05)$.

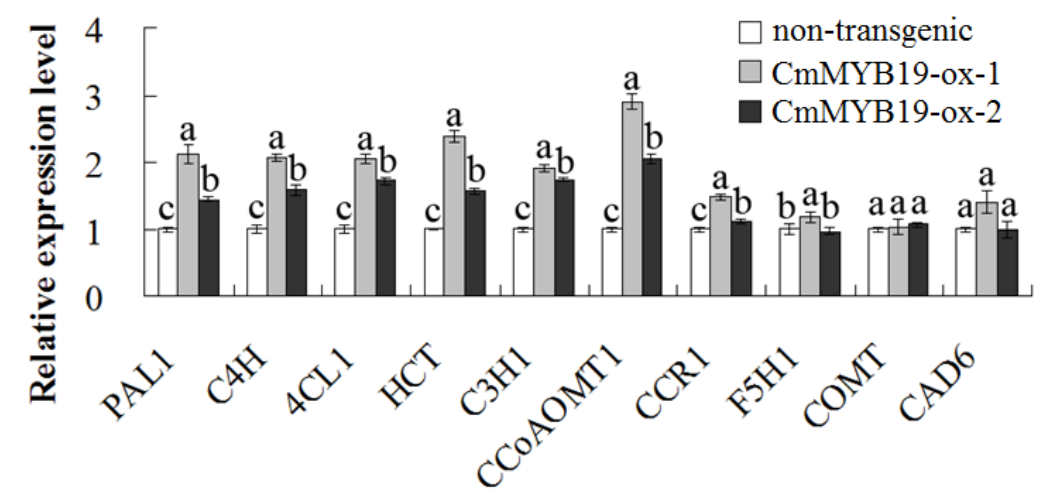

Figure 9. The transcription of genes involved in lignin synthesis in CmMYB19 over-expressing and non-transgenic plants, as assayed by qRT-PCR. For each gene, different letters $(a, b, c)$ represent significantly different expression levels between non-transgenic and CmMYB19 overexpressing plants $(p<0.05)$.

\section{Discussion}

Numerous studies have been performed to understand the functions of members of the $M Y B$ gene family previously [1]. However, rather few data have been generated in chrysanthemum. Two chrysanthemum MYB genes have been isolated so far. One of these, CmMYB1, when constitutively expressed in A. thaliana, has a suppressive effect on tissue lignin content and flavonoid synthesis [14], while the other (CmMYB2) not only enhances drought and salinity tolerance, but also increases the plant's sensitivity to abscisic acid and defers flowering [15]. Here, the R2R3-MYB member CmMYB19 was shown to be inducible by aphid feeding and appears to contribute to host tolerance against aphid feeding. 
The involvement of several A. thaliana $M Y B$ genes in the host response to aphid feeding have been reported in the literature. These include $A t M Y B 44$, which regulates tolerance to the green peach aphid and diamondback moth by activating the EIN2-mediated defense pathway [13]. Both AtMYB15 and AtMYB38 interact with a harpin protein to modulate the plant's tolerance to infestation by the green peach aphid [16]. The phylogenetic analysis indicated that CmMYB19 was included in the group of lignin biosynthesis or wall deposition [11], of which AtMYB103, AtMYB61, AtMYB55 [17] AtMYB83, AtMYB46 [18], AtMYB86 [19], AtMYB26 [20], and AtMYB50 [21] have been proved to be involved in the biosynthesis of lignin; however, the roles of AtMYB18, AtMYB19, AtMYB45 and AtMYB67 in lignin biosynthesis have not been described yet. Here, $C m M Y B 19$ overexpression enhanced the accumulation of lignin. Lignin is a vital component of the vascular plant secondary cell wall and contributes significantly to the erection of a physical barrier against invasion by various pathogens and feeding by various insects [22]. He et al. [23] have shown that the activity of PAL (an enzyme involved in lignin synthesis) is induced in chrysanthemum by aphid feeding, which implies a contribution of lignin to aphid tolerance. Aphids need to access the phloem for nutrient and to the xylem to avoid an excessive build-up of osmotic pressure resulting from their consumption of sugar-rich phloem fluid [24]. Thus, the great lignification of the CmMYB19 over-expressors may make it more difficult for the aphid stylet to gain access, and thus could act as the brake on aphid multiplication observed in the CmMYB19 over-expressors.

Lignin synthesis is regulated by a combination of MYB, NAC (NAM, ATAF1/2, CUC1/2) [8] and WRKY named after the highly conserved sequence motif WRKYGQK [25] transcription factors. AtMYB46 and AtMYB83 regulate the lignin pathway by combining with SND1, which holds a closely related NAC domain functioning to activate the entire secondary wall biosynthesis at the top of the network [26,27]. Populus trichocarpa PtrMYB152 is known to enhance secondary cell wall thickness by elevating lignification activity [28]. The constitutive expression of the Populus tomentosa MYB gene PtoMYB216 in A. thaliana activates the expression of a number of lignin synthesis genes, and results in the deposition of lignin even in cells which are normally non-lignified in non-transgenic Arabidopsis [6]. The promoter sequences of both PAL and $4 C L$ feature a high representation of AC elements. The promoters of the two Eucalyptus gunnii genes EgCCR and EgCAD2 each harbor a number of conserved MYB binding elements which are crucial for their transcriptional activation in the vascular tissue [29]. In Eriobotrya japonica, EjMYB1 and EjMYB2 regulate lignification in the fruit via a competitive interaction with AC elements in the promoter region of Ej4CL1 [30]. The Populus (Populus tremula $\times$ tremuloides) MYB46 homolog PttMYB021 activates the transcription in hybrid aspen of lignin synthesis enzymes and mediates the xylem-specific transcription of a number of secondary cell wall carbohydrate-active enzymes through its interaction with AC-type cis elements [31]. Here, one yeast hybrid assay suggested that CmMYB19 could bind to AC elements that are rich in lignin biosynthesis genes, suggesting that CmMYB19 functionally act as regulator of lignin biosynthesis (Supplemental Figure S3). Consistently, CmMYB19 over-expression was shown to upregulate a number of lignin synthesis genes (PAL1, C4H, 4CL1, HCT, C3H1, CCOAOMT1 and CCR1), if AC cis elements present in the promoters of any of these genes still remain to be investigated. Most MYB transcription factors appear to possess a transcriptional activation ability, although some only manifest this in the presence of specific bHLH proteins [32]. Here, CmMYB19 appeared to lack transcriptional activity in yeast, so whether CmMYB19 in vivo acts as an activator or whether it regulates lignin synthesis in combination with other protein(s) still need to be established. In the present study, a higher expression level of $C m M Y B 19$ in the leaves and a temporal variation in expression profiles of CmMYB19 in non-transgenic plants in the absence of aphid infestation such as at time points $3 \mathrm{~h}$ and $12 \mathrm{~h}$ suggested that $C m M Y B 19$ might participate in other biological processes besides regulating lignin biosynthesis. However, the exact processes are still unknown. Nevertheless, the present study inferred that CmMYB19 enhanced the aphid tolerance in chrysanthemum via increasing the lignin content. However, the two transgenic lines differed in behaviors of aphid tolerance and the induction of lignin gene expression to some extent, which might result from different spatial integration of CmMYB19 into 
the genome. Here, we successfully enhanced the aphid tolerance by modifying the physical defense. Alternative ways, for example, aimed to modify biosynthesis of toxins, and terpenes would obtain higher levels of tolerance or resistance to aphids.

\section{Materials and Methods}

\subsection{Plant Materials and Growing Conditions}

Cuttings of the chrysanthemum varieties "Nannong Xunzhang" (NX: aphid resistant, partially via up-regulating lignin biosynthesis) [33] and "Jinba" (JB: non-resistant) [23] were obtained from the Chrysanthemum Germplasm Resource Preserving Center (Nanjing Agricultural University, Nanjing, China). The cuttings were potted into a 1:2 mixture of vermiculite and garden soil and were maintained in a greenhouse providing a relative humidity of $80 \%$ and a $16 \mathrm{~h}$ photoperiod given by $100 \mu \mathrm{mol} \cdot \mathrm{m}^{-2} \cdot \mathrm{s}^{-1}$ of artificial light; the light and dark period temperatures were, respectively, $23^{\circ} \mathrm{C}$ and $18^{\circ} \mathrm{C}$.

\subsection{Isolation and Sequence Analysis of CmMYB19}

Total RNA was isolated from NX leaves using the RNAiso reagent (TaKaRa, Tokyo Japan) following the manufacturer's protocol. A $1 \mu \mathrm{g}$ aliquot of the resulting RNA was included as the template for first cDNA strand synthesis, using SuperScript III reverse transcriptase (Invitrogen, Carlsbad, CA, USA). The primer pair CmMYB19-M-F/R (Table S1) was designed to amplify a fragment of the CmMYB19 sequence, based on a previously acquired sequence [33], and RACE (random amplification of cDNA ends) PCR was then used to obtain the full length cDNA. For the $3^{\prime}$-RACE, oligo (dT) was used to synthesize the first cDNA strand, followed by a nested PCR using the adaptor primer (J-R) and CmMYB19-3-1/2 (Table S1). For the 5'-RACE, the AAP and AUAP primers provided with the 5'-RACE System kit v2.0 (Invitrogen) were used in a nested PCR, along with CmMYB19-5-1/2 (Table S1). The PCR products, purified using a Biospin Gel Extraction kit (Bio Flux, Hangzhou, China), were introduced into pMD19-T (TaKaRa) for sequencing. Finally, the CmMYB19 open reading frame (ORF) was amplified using CmMYB19-F/R (Table S1). The CmMYB19 protein sequence was aligned with its homologs using ClustalW software (available on: http://www.ebi.ac.uk/Tools/msa/clustalw2/) [34] and a phylogenetic tree was generated using MEGA 5 software (available on: http://www.megasoftware.net) [35] based on the neighbor-joining method and 1,000 bootstrap replicates following previous descriptions $[11,36]$. A. thaliana MYB polypeptides sequences were obtained from the Plant TFDB website (available on: planttfdb.cbi.edu.cn/).

\subsection{Transcriptional Activation Assay and Sub-Cellular Localization of CmMYB19}

The CmMYB19 ORF was amplified using a Phusion ${ }^{\circledR}$ High Fidelity PCR Kit (New England Biolabs, Ipswich, MA, USA), and KpnI and XhoI restriction sites were introduced with the primer pair CmMYB19-KPN-F/-XHO-R (Table S1). The resulting amplicon was digested by $\mathrm{KpnI} / \mathrm{XhoI}$, then ligated into $\mathrm{pENTR}^{\mathrm{TM}} 1 \mathrm{~A}$ (Invitrogen) to form the construct $p E^{\mathrm{N} T R^{\mathrm{TM}}} 1 A-C m M Y B 19$, which acts as a donor construct for yeast expressing construct and binary vector for chrysanthemum transformation. A number of clones were sequenced for validation purposes. For transcription activation ability assay of CmMYB19, GAL4/UAS system was used, where CmMYB19 was fused to GAL4 DNA biding domain using pDEST-GBKT7 vector [37]. To generate yeast expressing a construct pDEST-GBKT7-CmMYB19, CmMYB19 sequence was inserted into pDEST-GBKT7 via an LR (LR Clonase ${ }^{\mathrm{TM}}$ II enzyme mix (Invitrogen) reaction between pENTR ${ }^{\mathrm{TM}} 1 \mathrm{~A}-\mathrm{CmMYB19}$ and pDEST-GBKT7. The resulting construct pDEST-GBKT7-CmMYB19, pDEST-GBKT7 (negative control) and $p C L 1$ (positive control) were each introduced separately into Y2HGold yeast cells (Clontech, Mountain View, CA, USA), following the manufacturer's protocol [38]. Transformants harboring either pDEST-GBKT7-CmMYB19 or pDEST-GBKT7 were selected on SD/-Trp medium (SD, Synthetic 
Dropout Media), while those harboring $p C L 1$ were selected on SD/-Leu medium. Selected clones were then transferred to an $\mathrm{SD} /$-His-Ade medium and incubated for two to three days at $30^{\circ} \mathrm{C}$, transcription activity was determined when the clones could induce expression of reporter genes and grow on the SD/-His-Ade medium. The sub-cellular localization of CmMYB19 was identified by transiently transforming onion epidermal cells with the construct p35S::GFP-CmMYB19 (GFP, the green-fluorescent protein) generated by LR reaction between PENTR $^{\mathrm{TM}} 1 \mathrm{~A}-\mathrm{C} m M Y B 19$ and pMDC43 (Invitrogen) [39]. The p35S::GFP-CmMYB19 construct and the empty pMDC43 vector were introduced into onion epidermal cells via particle bombardment (PDS-1000; Bio-Rad, Tokyo, Japan). Transformed cells were held for $16 \mathrm{~h}$ on Murashige and Skoog (MS) medium in the dark, and then subjected to confocal laser microscopy in order to monitor GFP activity [40].

\subsection{Transcription of CmMYB19 and Its Response to Aphid Infestation}

Roots, stems and leaves were harvested from four week old NX plants to characterize the topological profile of CmMYB19 transcription. To investigate the response to aphid (M. sanborni) feeding, a phenotyptically uniform set of 8-10 leaf stage NX plants were each infested with $20 \mathrm{~s}$ instar nymphs. The nymphs were placed onto the third fully expanded leaf (counting from the stem apex) using a soft brush. The inoculated leaves were enclosed within a transparent, ventilated plastic cage. The leaves of three inoculated plants were harvested at $-2 \mathrm{~h}, 0 \mathrm{~h}, 3 \mathrm{~h}, 6 \mathrm{~h}, 9 \mathrm{~h}, 12 \mathrm{~h}, 24 \mathrm{~h}$ (where $0 \mathrm{~h}$ refers to $2 \mathrm{~h}$ after the infestation), following Xia et al. [33]. As a control to account for any stress imposed by aphid stylet penetration, a mock puncture treatment was imposed, in which leaves were pricked with a needle five times at $0 \mathrm{~h}$, ten times at both $3 \mathrm{~h}$ and $6 \mathrm{~h}, 15$ times at $9 \mathrm{~h}$ and $12 \mathrm{~h}$ and 20 times at $24 \mathrm{~h} \mathrm{[41].} \mathrm{Each} \mathrm{experiment} \mathrm{was} \mathrm{replicated} \mathrm{three} \mathrm{times.} \mathrm{Leaf} \mathrm{samples} \mathrm{required} \mathrm{for} \mathrm{RNA}$ extraction were snap-frozen in liquid nitrogen and stored at $-80{ }^{\circ} \mathrm{C}$. Total RNA (isolated as described earlier) was treated with RNase-free DNase I (TaKaRa), and then $1 \mu \mathrm{g}$ treated RNA was converted into cDNA using M-MLV (MLV, clone 3 Moloney-murine leukemia virus) reverse transcriptase (TaKaRa) following the manufacturer's protocol. Transcript abundance was detected by quantitative real-time PCR (qRT-PCR). The reactions contained SYBR ${ }^{\circledR}$ Premix Ex Taq ${ }^{\text {TM }}$ II (Tli RNaseH Plus) (TaKaRa) and the primer pair CmMYB19-RT-F/R (Table S1). The primer pair CmEF1á-F/R was used to amplify the reference gene CmEF1á. Fold changes were calculated using the $2^{-\Delta \Delta C t}$ method [42]. The experiment included three biological replicates. The statistics was calculated from three biological replicates.

\subsection{Generation of Transgenic Chrysanthemum and the Determination of Aphid Tolerance}

CmMYB19 over-expressors in a JB background were generated by leaf disc agroinfection, based on Agrobacterium tumefaciens strain EHA105 [43]. RNA was extracted (as described in an earlier section) from both putative $p 35:: C m M Y B 19$-harboring and WT (the non-transgenic chrysanthemum 'Jinba') plants. CmMYB19 transcript abundance was detected using qRT-PCR. Five second instar M. sanborni nymphs were placed on both WT plants and two independent over-expressing lines. Each plant was then enclosed in a $25 \mathrm{~cm} \times 12 \mathrm{~cm}$ transparent plastic cylinder capped with gauze [38]. Aphid numbers were counted every two days starting three days after the initial aphid infestation (DAI) and ending after 21 days. The experiment involved three replicates of 20 plants per each tested line. The aphid numbers were used to calculate both a multiplication rate (MR) and an inhibition ratio (IR), where MR was given by the number of aphids present at 21 DAI divided by five. IR was calculated from the ratio $\left(N_{\mathrm{W}}-N_{\mathrm{T}}\right) / N_{\mathrm{W}}$, where $N_{\mathrm{W}}$ and $N_{\mathrm{T}}$ represented the number of aphids present at $21 \mathrm{DAI}$ on, respectively, $\mathrm{WT}$ and the over-expressing plants.

\subsection{Determination of Lignin Content and the Transcription of Lignin Synthesis Genes}

For lignin content analysis, stems from WT and CmMYB19 over-expressing plants at the 6-8 leaf stage were harvested, lignin content was determined using the acetyl bromide method [44]. Briefly, harvested stems were dried at $60^{\circ} \mathrm{C}$ for $48 \mathrm{~h}$. In addition, $20 \mathrm{mg}$ dried samples were sonicated with $5 \mathrm{~mL}$ acetone for $30 \mathrm{~min}$ in a 10-mL screw-capped test tube. The supernant extract was pipetted off, 
and the extractive free sample was dried, and redissolved in $5 \mathrm{~mL} \mathrm{20 \%}(v / v)$ AcBr-acetic acid solution containing $100 \mu \mathrm{L} 70 \%$ perchloric acid and the sample was kept in a block heater at $50{ }^{\circ} \mathrm{C}$ for $3 \mathrm{~h}$ with regular shaking. The reaction was stopped by keeping the samples at $-20^{\circ} \mathrm{C}$ for $15 \mathrm{~min}$. The solution was transferred to a 5-mL screw-capped test tube containing $1 \mathrm{~mL} 2 \mathrm{M} \mathrm{NaOH}$ and $0.1 \mathrm{~mL}$ freshly prepared $0.5 \mathrm{M}$ hydroxylamine hydrochloride, filled up to $5 \mathrm{~mL}$ with glacial acetic acid, and inverted several times in order to mix. The UV spectrum was measured with a Shimadzu UV-2401 spectrometer (Shimadzu Corp, Kyoto, Japan) at $280 \mathrm{~nm}$. Lignin content was calculated using the following expression: Lignin $\%=100(\mathrm{As}-\mathrm{Ab}) \mathrm{V} / a \mathrm{~W}$, where As represents absorbance of sample; $\mathrm{Ab}$ is absorbance of blank; $\mathrm{V}$ represents the volume of solution; $\mathrm{W}$ is weight of sample; and $a$ is the absorptivity of a lignin standard calculated for each analysis series. The transcript abundances of CmPAL1, CmC4H, Cm4CL1, CmHCT, CmC3H1, CmCCoAOMT1, CmCCR1, CmF5H1, CmCOMT and CmCAD6 were detected using qRT-PCR, as described above, with relevant primer sequences given in Table S1. The template for the qRT-PCRs was total RNA prepared, as described above, from the stems of both WT and CmMYB19 over-expressing plants. The relative expression level of a gene is the fold change in expression of a gene in a sample compared to the calibrator sample. The expression level of CmMYB19 in the root, that at " $-2 \mathrm{~h}$ " time point of control, and of non-transgenic plants, were set as calibrator " 1 ", respectively. The experiment included three biological replicates.

\subsection{Statistical Analysis}

Tukey's multiple range test $(p=0.05)$ was used to compare means. Calculations were performed by routines implemented in SPSS 16.0 software (SPSS Inc., Chicago, IL, USA).

\section{Conclusions}

Taken together, MYB transcription factor CmMYB19 from chrysanthemum encodes a R2R3-MYB. It localized in the nuclei, and expresses no transcriptional activation in yeast cells. CmMYB19 transcription was induced by aphid infestation. The over-expression of CmMYB19 restricted the multiplication of the aphids on the host, mediated by an enhanced accumulation of lignin.

Supplementary Materials: Supplementary materials can be found at www.mdpi.com/1422-0067/18/3/619/s1.

Acknowledgments: The work was funded by the National Natural Science Foundation of China (Grant Nos. 31672192, 31471913, 31272202, 31471900), the Fundamental Research Funds for the Central Universities (Grant No. KYTZ201401) and the Project Funded by the Priority Academic Program Development of Jiangsu Higher Education Institutions.

Author Contributions: Liping Sheng and Sumei Chen conceived and designed the experiments. Liping Sheng, Yinjie Wang and Xinping Du performed the experiments. Liping Sheng, Huanru Zhang, Xiaolong Xia and Cong An analyzed the data. Jiafu Jiang and Fadi Chen contributed reagents/plant materials/analysis tools. Liping Sheng and Sumei Chen wrote the manuscript. All authors read and approved the manuscript.

Conflicts of Interest: The authors declare that they have no conflict of interest.

\section{References}

1. Ambawat, S.; Sharma, P.; Yadav, N.R.; Yadav, R.C. MYB transcription factor genes as regulators for plant responses: An overview. Physiol. Mol. Biol. Plants 2013, 19, 307-321. [CrossRef] [PubMed]

2. Du, H.; Zhang, L.; Liu, L.; Tang, X.-F.; Yang, W.-J.; Wu, Y.-M.; Huang, Y.-B.; Tang, Y.-X. Biochemical and molecular characterization of plant MYB transcription factor family. Biochemistry 2009, 74, 1-11. [CrossRef] [PubMed]

3. Dubos, C.; Stracke, R.; Grotewold, E.; Weisshaar, B.; Martin, C.; Lepiniec, L. MYB transcription factors in Arabidopsis. Trends Plant Sci. 2010, 15, 573-581. [CrossRef] [PubMed]

4. Zhou, J.; Lee, C.; Zhong, R.; Ye, Z.-H. MYB58 and MYB63 are transcriptional activators of the lignin biosynthetic pathway during secondary cell wall formation in Arabidopsis. Plant Cell 2009, 21, $248-266$. [CrossRef] [PubMed] 
5. Zhong, R.; Richardson, E.A.; Ye, Z.-H. The MYB46 transcription factor is a direct target of SND1 and regulates secondary wall biosynthesis in Arabidopsis. Plant Cell 2007, 19, 2776-2792. [CrossRef] [PubMed]

6. Tian, Q.; Wang, X.; Li, C.; Lu, W.; Yang, L.; Jiang, Y.; Luo, K. Functional characterization of the poplar R2R3-MYB transcription factor PtoMYB216 involved in the regulation of lignin biosynthesis during wood formation. PLoS ONE 2013, 8, e76369. [CrossRef] [PubMed]

7. Tang, X.; Zhuang, Y.; Qi, G.; Wang, D.; Liu, H.; Wang, K.; Chai, G.; Zhou, G. Poplar PdMYB221 is involved in the direct and indirect regulation of secondary wall biosynthesis during wood formation. Sci. Rep. 2015. [CrossRef] [PubMed]

8. Zhao, Q.; Dixon, R.A. Transcriptional networks for lignin biosynthesis: More complex than we thought? Trends Plant Sci. 2011, 16, 227-233. [CrossRef] [PubMed]

9. Deng, Y.; Chen, S.; Lu, A.; Chen, F.; Tang, F.; Guan, Z.; Teng, N. Production and characterisation of the intergeneric hybrids between Dendranthema morifolium and Artemisia vulgaris exhibiting enhanced resistance to chrysanthemum aphid (Macrosiphoniella sanbourni). Planta 2010, 231, 693-703. [CrossRef] [PubMed]

10. Goggin, F.L. Plant-aphid interactions: Molecular and ecological perspectives. Curr. Opin. Plant Biol. 2007, 10, 399-408. [CrossRef] [PubMed]

11. Chen, N.; Yang, Q.; Pan, L.; Chi, X.; Chen, M.; Hu, D.; Yang, Z.; Wang, T.; Wang, M.; Yu, S. Identification of 30 MYB transcription factor genes and analysis of their expression during abiotic stress in peanut (Arachis hypogaea L.). Gene 2014, 533, 332-345. [CrossRef] [PubMed]

12. De Vos, M.; Denekamp, M.; Dicke, M.; Vuylsteke, M.; Van Loon, L.; Smeekens, S.C.; Pieterse, C. The Arabidopsis thaliana transcription factor AtMYB102 functions in defense against the insect herbivore Pieris rapae. Plant Signal. Behav. 2006, 1, 305-311. [CrossRef] [PubMed]

13. Lü, B.B.; Li, X.J.; Sun, W.W.; Li, L.; Gao, R.; Zhu, Q.; Tian, S.M.; Fu, M.Q.; Yu, H.L.; Tang, X.M. AtMYB44 regulates resistance to the green peach aphid and diamondback moth by activating EIN2-affected defences in Arabidopsis. Plant Biol. 2013, 15, 841-850. [CrossRef] [PubMed]

14. Zhu, L.; Shan, H.; Chen, S.; Jiang, J.; Gu, C.; Zhou, G.; Chen, Y.; Song, A.; Chen, F. The heterologous expression of the chrysanthemum R2R3-MYB transcription factor CmMYB1 alters lignin composition and represses flavonoid synthesis in Arabidopsis thaliana. PLoS ONE 2013, 8, e65680. [CrossRef] [PubMed]

15. Shan, H.; Chen, S.; Jiang, J.; Chen, F.; Chen, Y.; Gu, C.; Li, P.; Song, A.; Zhu, X.; Gao, H. Heterologous expression of the chrysanthemum R2R3-MYB transcription factor CmMYB2 enhances drought and salinity tolerance, increases hypersensitivity to ABA and delays flowering in Arabidopsis thaliana. Mol. Biotechnol. 2012, 51, 160-173. [CrossRef] [PubMed]

16. Liu, R.; Lü, B.; Wang, X.; Zhang, C.; Zhang, S.; Qian, J.; Chen, L.; Shi, H.; Dong, H. Thirty-seven transcription factor genes differentially respond to a harpin protein and affect resistance to the green peach aphid in Arabidopsis. J. Biosci. 2010, 35, 435-450. [CrossRef] [PubMed]

17. Hirano, K.; Kondo, M.; Aya, K.; Miyao, A.; Sato, Y.; Antonio, B.A.; Namiki, N.; Nagamura, Y.; Matsuoka, M. Identification of transcription factors involved in rice secondary cell wall formation. Plant Cell Physiol. 2013, 54, 1791-1802. [CrossRef] [PubMed]

18. Öhman, D.; Demedts, B.; Kumar, M.; Gerber, L.; Gorzsás, A.; Goeminne, G.; Hedenström, M.; Ellis, B.; Boerjan, W.; Sundberg, B. MYB103 is required for FERULATE-5-HYDROXYLASE expression and syringyl lignin biosynthesis in Arabidopsis stems. Plant J. 2013, 73, 63-76. [CrossRef] [PubMed]

19. Zhong, R.; Lee, C.; Zhou, J.; McCarthy, R.L.; Ye, Z.-H. A battery of transcription factors involved in the regulation of secondary cell wall biosynthesis in Arabidopsis. Plant Cell 2008, 20, 2763-2782. [CrossRef] [PubMed]

20. Yang, C.; Xu, Z.; Song, J.; Conner, K.; Barrena, G.V.; Wilson, Z.A. Arabidopsis MYB26/MALE STERILE35 regulates secondary thickening in the endothecium and is essential for anther dehiscence. Plant Cell 2007, 19, 534-548. [CrossRef] [PubMed]

21. Guo, K.; Zou, W.; Feng, Y.; Zhang, M.; Zhang, J.; Tu, F.; Xie, G.; Wang, L.; Wang, Y.; Klie, S. An integrated genomic and metabolomic framework for cell wall biology in rice. BMC Genom. 2014, 15, 596. [CrossRef] [PubMed] 
22. Chantreau, M.; Portelette, A.; Dauwe, R.; Kiyoto, S.; Crônier, D.; Morreel, K.; Arribat, S.; Neutelings, G.; Chabi, M.; Boerjan, W. Ectopic lignification in the flax lignified bast fiber1 mutant stem is associated with tissue-specific modifications in gene expression and cell wall composition. Plant Cell 2014, 26, 4462-4482. [CrossRef] [PubMed]

23. He, J.; Chen, F.; Chen, S.; Lv, G.; Deng, Y.; Fang, W.; Liu, Z.; Guan, Z.; He, C. Chrysanthemum leaf epidermal surface morphology and antioxidant and defense enzyme activity in response to aphid infestation. J. Plant Physiol. 2011, 168, 687-693. [CrossRef] [PubMed]

24. Guo, H.; Sun, Y.; Peng, X.; Wang, Q.; Harris, M.; Ge, F. Up-regulation of abscisic acid signaling pathway facilitates aphid xylem absorption and osmoregulation under drought stress. J. Exp. Bot. 2016, 67, 681-693. [CrossRef] [PubMed]

25. Wang, H.; Avci, U.; Nakashima, J.; Hahn, M.G.; Chen, F.; Dixon, R.A. Mutation of WRKY transcription factors initiates pith secondary wall formation and increases stem biomass in dicotyledonous plants. Proc. Natl. Acad. Sci. USA 2010, 107, 22338-22343. [CrossRef] [PubMed]

26. McCarthy, R.L.; Zhong, R.; Ye, Z.-H. MYB83 is a direct target of SND1 and acts redundantly with MYB46 in the regulation of secondary cell wall biosynthesis in Arabidopsis. Plant Cell Physiol. 2009, 50, 1950-1964. [CrossRef] [PubMed]

27. Zhong, R.; Ye, Z.-H. MYB46 and MYB83 bind to the SMRE sites and directly activate a suite of transcription factors and secondary wall biosynthetic genes. Plant Cell Physiol. 2012, 53, 368-380. [CrossRef] [PubMed]

28. Wang, S.; Li, E.; Porth, I.; Chen, J.-G.; Mansfield, S.D.; Douglas, C.J. Regulation of secondary cell wall biosynthesis by poplar R2R3 MYB transcription factor PtrMYB152 in Arabidopsis. Sci. Rep. 2014. [CrossRef] [PubMed]

29. Rahantamalala, A.; Rech, P.; Martinez, Y.; Chaubet-Gigot, N.; Grima-Pettenati, J.; Pacquit, V. Coordinated transcriptional regulation of two key genes in the lignin branch pathway-CAD and CCR-is mediated through MYB-binding sites. BMC Plant Biol. 2010, 10, 130. [CrossRef] [PubMed]

30. Xu, Q.; Yin, X.-R.; Zeng, J.-K.; Ge, H.; Song, M.; Xu, C.-J.; Li, X.; Ferguson, I.B.; Chen, K.-S. Activator-and repressor-type MYB transcription factors are involved in chilling injury induced flesh lignification in loquat via their interactions with the phenylpropanoid pathway. J. Exp. Bot. 2014, 65, 4349-4359. [CrossRef] [PubMed]

31. Winzell, A.; Aspeborg, H.; Wang, Y.; Ezcurra, I. Conserved CA-rich motifs in gene promoters of Pt $\times$ tMYB021-responsive secondary cell wall carbohydrate-active enzymes in Populus. Biochem. Biophys. Res. Commun. 2010, 394, 848-853. [CrossRef] [PubMed]

32. Nesi, N.; Jond, C.; Debeaujon, I.; Caboche, M.; Lepiniec, L. The Arabidopsis TT2 gene encodes an R2R3 MYB domain protein that acts as a key determinant for proanthocyanidin accumulation in developing seed. Plant Cell 2001, 13, 2099-2114. [PubMed]

33. Xia, X.; Shao, Y.; Jiang, J.; Ren, L.; Chen, F.; Fang, W.; Guan, Z.; Chen, S. Gene expression profiles responses to aphid feeding in chrysanthemum (Chrysanthemum morifolium). BMC Genom. 2014, 15, 1. [CrossRef] [PubMed]

34. Larkin, M.A.; Blackshields, G.; Brown, N.; Chenna, R.; McGettigan, P.A.; McWilliam, H.; Valentin, F.; Wallace, I.M.; Wilm, A.; Lopez, R. Clustal W and Clustal X version 2.0. Bioinformatics 2007, 23, 2947-2948. [CrossRef] [PubMed]

35. Tamura, K.; Peterson, D.; Peterson, N.; Stecher, G.; Nei, M.; Kumar, S. MEGA5: Molecular evolutionary genetics analysis using maximum likelihood, evolutionary distance, and maximum parsimony methods. Mol. Biol. Evol. 2011, 28, 2731-2739. [CrossRef] [PubMed]

36. Li, P.; Wu, Z.; Liu, T.; Wang, Y. Biodiversity, Phylogeny, and Antifungal Functions of Endophytic Fungi Associated with Zanthoxylum bungeanum. Int. J. Mol. Sci. 2016, 17, 1541. [CrossRef] [PubMed]

37. Gao, H.; Song, A.; Zhu, X.; Chen, F.; Jiang, J.; Chen, Y.; Sun, Y.; Shan, H.; Gu, C.; Li, P. The heterologous expression in Arabidopsis of a chrysanthemum Cys2/His2 zinc finger protein gene confers salinity and drought tolerance. Planta 2012, 235, 979-993. [CrossRef] [PubMed]

38. Li, P.; Song, A.; Gao, C.; Jiang, J.; Chen, S.; Fang, W.; Zhang, F.; Chen, F. The over-expression of a chrysanthemum WRKY transcription factor enhances aphid resistance. Plant Physiol. Biochem. 2015, 95, 26-34. [CrossRef] [PubMed]

39. Fan, Q.; Song, A.; Xin, J.; Chen, S.; Jiang, J.; Wang, Y.; Li, X.; Chen, F. CmWRKY15 facilitates Alternaria tenuissima infection of chrysanthemum. PLOS ONE 2015, 10, e0143349. [CrossRef] [PubMed] 
40. Jaffar, M.A.; Song, A.; Faheem, M.; Chen, S.; Jiang, J.; Liu, C.; Fan, Q.; Chen, F. Involvement of CmWRKY10 in drought tolerance of chrysanthemum through the ABA-signaling pathway. Int. J. Mol. Sci. 2016, 17, 693. [CrossRef] [PubMed]

41. Moran, P.J.; Thompson, G.A. Molecular responses to aphid feeding in Arabidopsis in relation to plant defense pathways. Plant Physiol. 2001, 125, 1074-1085. [CrossRef] [PubMed]

42. Livak, K.J.; Schmittgen, T.D. Analysis of relative gene expression data using real-time quantitative PCR and the 2- $\Delta \Delta C$ T method. Methods 2001, 25, 402-408. [CrossRef] [PubMed]

43. Liu, P.; Chen, S.; Song, A.; Zhao, S.; Fang, W.; Guan, Z.; Liao, Y.; Jiang, J.; Chen, F. A putative high affinity phosphate transporter, CmPT1, enhances tolerance to Pi deficiency of chrysanthemum. BMC Plant Biol. 2014, 14, 1. [CrossRef] [PubMed]

44. Fagerstedt, K.V.; Saranpää, P.; Tapanila, T.; Immanen, J.; Serra, J.A. A.; Nieminen, K. Determining the composition of lignins in different tissues of silver birch. Plants 2015, 4, 183-195. [CrossRef] [PubMed]

(c) 2017 by the authors. Licensee MDPI, Basel, Switzerland. This article is an open access article distributed under the terms and conditions of the Creative Commons Attribution (CC BY) license (http://creativecommons.org/licenses/by/4.0/). 